\title{
ON THE DYNAMIC PROPERTIES OF FLEXIBLE PARALLEL MANIPULATORS IN THE PRESENCE OF PAYLOAD AND TYPE 2 SINGULARITIES
}

\author{
Sébastien Briot ${ }^{\mathrm{a}, *}$ \\ Vigen Arakelian ${ }^{\mathrm{b}}$ \\ ${ }^{a}$ Institut de Recherches en Communications et Cybernétique \\ de Nantes (IRCCyN), UMR CNRS 6597 \\ 1 rue de la Noë, BP 92101, 44321 Nantes Cedex 3, France \\ ${ }^{\mathrm{b}}$ Département de Génie Mécanique et Automatique - L.G.C.G.M. EA3913 \\ Institut National des Sciences Appliquées (I.N.S.A.) \\ 20 avenue des Buttes de Coësmes - CS 14315 \\ F-35043 Rennes, France
}

\begin{abstract}
It is known that a parallel manipulator at a singular configuration can gain one or more degrees of freedom and become uncontrollable. In our recent work [1], the dynamic properties of rigid-link parallel manipulators, in the presence of Type 2 singularities, have been studied. It was shown that any parallel manipulator can pass through the singular positions without perturbation of motion if the wrench applied on the end-effector by the legs and external efforts is orthogonal to the twist along the direction of the uncontrollable motion. This condition was obtained using symbolic approach based on the inverse dynamics and the study of the Lagrangian of a general rigid-link parallel manipulator. It was validated by experimental tests carried out on the prototype of a four-degrees-of-freedom parallel manipulator. However, it is known that the flexibility of the mechanism may not always been neglected. Indeed, for robots, joint flexibility can be the main source contributing to overall manipulator flexibility and can lead to trajectory distortion. Therefore, in our second paper [2], the condition of passing through a Type 2 singularity for parallel manipulators with flexible joints has been studied.

In the present paper, we expand information about the dynamic properties of parallel manipulators in the presence of Type 2 singularity by including in the studied problem the link flexibility and the payload. The suggested technique is illustrated by a $5 R$ parallel manipulator with flexible elements (actuated joints and moving links) and a payload. The
\end{abstract}

obtained results are validated by numerical simulations carried out using the software ADAMS.

\section{INTRODUCTION}

There are many papers dealing with the singularity analysis of parallel manipulators. We present some of them in the remaining.

The analysis of singular configurations has been first discussed from a kinematic point of view [3]-[12]. However, it is also known that, when parallel manipulators have Type 2 singularities [3], they lose their stiffness and their quality of motion transmission, and as a result, their payload capability. Therefore, the singularity zones in the workspace of manipulators may be analyzed not only in terms of kinematic criterions, from the theoretically perfect model of manipulators, but also in terms of kinetostatic approaches [13]-[19].

The further study of singularity in parallel manipulators has revealed an interesting problem that concerns the path planning of parallel manipulators under the presence of singular positions, i.e. the motion feasibility in the neighbourhood of singularities. In this case the dynamic conditions can be considered in the path planning process. One of the most evident solutions for the stable motion generation in the neighbourhood of singularities is to use redundant sensors and actuators [20]-[24]. However, it is an expensive solution to the problem because of the additional actuators and the complicated control of the manipulator caused by actuation redundancy. Another approach concerns with motion planning to pass through singularity [25]-[30],

\footnotetext{
*Author of correspondence, Phone: +33 2403769 58, Fax: +33 24037 69 30, Email: Sebastien.Briot@irccyn.ec-nantes.fr.
} 
i.e. a parallel manipulator may track a path through singular poses if its velocity and acceleration are properly constrained. This is a promising way for the solution of this problem. However, only a few research papers on this approach have addressed the path planning for obtaining a good tracking performance. But they have not adequately addressed the physical interpretation of dynamic aspects.

In [1], optimal force generation in parallel manipulators for passing through the singular positions has been studied. It was shown that any parallel manipulator can pass through the singular positions without perturbation of motion if the wrench applied on the end-effector by the legs and external efforts of the manipulator are orthogonal to the twist along the direction of the uncontrollable motion. This approach has been generalised in the case of rigid-link flexible-joints parallel manipulators [2].

This study is the continuation of works [1], [2]. The purpose of this paper is to study the dynamic properties of parallel manipulators with flexible links and joints in the presence of preponderant payload, which can be the main source of elastic deformations.

The paper is organized as follows. The next section presents theoretical aspects of the examined problem, using the Lagrangian formulation. The condition of force distribution is defined, that allows the passing of any parallel manipulator through the Type 2 singular positions. In section 3 , the suggested solution is illustrated via $5 R$ planar parallel manipulator. In section 4, the conclusions are given.

\section{OPTIMAL DYNAMIC CONDITIONS FOR PASSING THROUGH TYPE 2 SINGULARITY}

Let us consider a parallel manipulator of $m$ links, $n$ degrees of freedom and driven by $n$ actuators, for which the effects of the payload and of the geared motors inertia are preponderant from the others.

The general Lagrangian dynamic formulation for a nonrigid manipulator can be expressed as [31]:

$$
\begin{gathered}
\boldsymbol{\tau}=\frac{\mathrm{d}}{\mathrm{dt}}\left(\frac{\partial L}{\partial \dot{\mathbf{q}}_{a}}\right)-\frac{\partial L}{\partial \mathbf{q}_{a}}, \\
\mathbf{0}=\frac{\mathrm{d}}{\mathrm{dt}}\left(\frac{\partial L}{\partial \dot{\mathbf{q}}_{e}}\right)-\frac{\partial L}{\partial \mathbf{q}_{e}},
\end{gathered}
$$

where,

- $L$ is the Lagrangian of the manipulator; $L=T-V$, where $T$ is the kinetic energy and $V$ the potential energy due to gravitational forces, friction and elasticity;

- $\mathbf{q}_{a}=\left[q_{1}^{a}, q_{2}^{a}, \ldots, q_{n}^{a}\right]^{T}$ and $\dot{\mathbf{q}}_{a}=\left[\dot{q}_{1}^{a}, \dot{q}_{2}^{a}, \ldots, \dot{q}_{n}^{a}\right]^{T}$ represent the vectors of position and velocity of the actuators, respectively;

- $\mathbf{q}_{e}=\left[q_{1}^{e}, q_{2}^{e}, \ldots, q_{n}^{e}\right]^{T}$ and $\dot{\mathbf{q}}_{e}=\left[\dot{q}_{1}^{e}, \dot{q}_{2}^{e}, \ldots, \dot{q}_{n}^{e}\right]^{T}$ represent the vectors of position and velocity of the elastic coordinates (deformations of links and joints);

- $\tau$ is the vector of the actuators efforts.

In the case where the load on the end-effector and the inertia of actuators are preponderant, the expressions of the kinetic energy $T$ and potential energy $V$ are given by:

$$
\begin{gathered}
2 T=\mathbf{v}^{T} \mathbf{M} \mathbf{v}+\dot{\mathbf{q}}_{a}^{T} \mathbf{I}_{a} \dot{\mathbf{q}}_{a}, \\
V=V_{g}+V_{e}, V_{g}=m g z \text { and } V_{e}=f\left(\mathbf{q}_{e}\right)
\end{gathered}
$$

where $V_{g}$ corresponds to the potential energy due to gravity, and $V_{e}$ to the energy due to elastic deformations. $\mathbf{M}$ is the mass matrix of the payload (comprising both mass and rotational inertia terms), $\mathbf{I}_{a}$ the diagonal matrix containing the axial moments of inertia of the geared motors, $m$ the mass of the load, $g$ the gravity, $f\left(\mathbf{q}_{e}\right)$ being a function depending of the deformations $\quad \mathbf{q}_{e}$. Moreover, $\quad \mathbf{x}=[x, y, z, \phi, \psi, \theta]^{T} \quad$ and $\mathbf{v}=[\dot{x}, \dot{y}, \dot{z}, \dot{\phi}, \dot{\psi}, \dot{\theta}]^{T}$ are vectors containing the trajectory parameters and their derivatives, respectively; $x, y, z$ represent the position of the controlled point in the global frame and $\phi, \psi$ and $\theta$ the rotation of the platform about three axes $\mathbf{a}_{\phi}$, $\mathbf{a}_{\psi}$ and $\mathbf{a}_{\theta}$. Vector $\mathbf{x}$ depends on both rigid coordinates $\mathbf{q}_{a}$ and elastic coordinates $\mathbf{q}_{e}$.

Analyzing expression (2), the potential and kinetic energies do not explicitly depend both of the actuated variables $\mathbf{q}_{a}$ and elastic coordinates $\mathbf{q}_{e}$, but also from the positions $\mathbf{x}$ and velocities $\mathbf{v}$ of the payload. Therefore it is preferable to rewrite Eq. (1) using the Lagrange multipliers [31], as follows:

$$
\begin{gathered}
\boldsymbol{\tau}=\mathbf{W}_{\mathbf{b}}+\mathbf{B}^{T} \boldsymbol{\lambda}, \mathbf{W}_{\mathbf{b}}=\frac{\mathrm{d}}{\mathrm{dt}}\left(\frac{\partial L}{\partial \dot{\mathbf{q}}_{a}}\right)-\frac{\partial L}{\partial \mathbf{q}_{a}}=\mathbf{I}_{a} \ddot{\mathbf{q}}_{a} \\
\mathbf{0}=\mathbf{W}_{\mathbf{c}}+\mathbf{C}^{T} \boldsymbol{\lambda}, \mathbf{W}_{\mathbf{c}}=\frac{\mathrm{d}}{\mathrm{dt}}\left(\frac{\partial L}{\partial \dot{\mathbf{q}}_{e}}\right)-\frac{\partial L}{\partial \mathbf{q}_{e}}
\end{gathered}
$$

where $\lambda$ is the Lagrange multipliers vector, which is related to the wrench $\mathbf{W}_{\mathbf{p}}$ applied on the platform by:

$$
\mathbf{A}^{T} \boldsymbol{\lambda}=\mathbf{W}_{\mathbf{p}}, \mathbf{W}_{\mathbf{p}}=\left(\frac{\mathrm{d}}{\mathrm{dt}}\left(\frac{\partial L}{\partial \mathbf{v}}\right)-\frac{\partial L}{\partial \mathbf{x}}\right)
$$

and,

- A, B and $\mathbf{C}$ are three matrices relating the vectors $\mathbf{v}, \dot{\mathbf{q}}_{e}$ and $\dot{\mathbf{q}}_{a}$ according to $\mathbf{A v}=\mathbf{B} \dot{\mathbf{q}}_{a}+\mathbf{C} \dot{\mathbf{q}}_{e}$. They can be found by differentiating the closure equations $f_{i}\left(\mathbf{x}, \mathbf{q}_{a}, \mathbf{q}_{e}\right)=0$ (taking into account the rigid as well as the elastic coordinates [31]) with respect to time. In the hypothesis of small elastic displacements $\left(\mathbf{q}_{e} \approx \mathbf{0}\right)$, matrices $\mathbf{A}$ and $\mathbf{B}$ may be found assuming that the robot is composed of rigid links only.

- $\mathbf{W}_{\mathbf{p}}$ is the wrench applied on the platform by the legs and external forces expressed along axes $\mathbf{a}_{\phi}, \mathbf{a}_{\psi}$ and $\mathbf{a}_{\theta}$ [32].

Expressing $\mathbf{W}_{\mathbf{p}}$ in the base frame, one can obtain:

$$
\begin{gathered}
\boldsymbol{\tau}=\mathbf{I}_{a} \ddot{\mathbf{q}}_{a}+\mathbf{J}_{\mathbf{q}_{a}}^{T} \mathbf{R}_{0} \mathbf{W}_{\mathbf{p}}, \\
\mathbf{0}=\mathbf{W}_{\mathbf{c}}+\mathbf{J}_{\mathbf{q}_{e}}^{T} \mathbf{R}_{0} \mathbf{W}_{\mathbf{p}},
\end{gathered}
$$

where $\mathbf{J}_{\mathbf{q}_{a}}=\left({ }^{\mathbf{R}_{0}} \mathbf{A}\right)^{-1} \mathbf{B}$ is the square Jacobian matrix between twist $\mathbf{t}$ of the platform (expressed in the base frame) and the vector $\dot{\mathbf{q}}_{a}$ of actuators velocities, $\mathbf{J}_{\mathbf{q}_{e}}=\left({ }^{\mathbf{R}_{0}} \mathbf{A}\right)^{-1} \mathbf{C}$ is the nonsquare Jacobian matrix between twist $\mathbf{t}$ of the platform (expressed in the base frame) and the vector $\dot{\mathbf{q}}_{e}$ of deformations velocities, ${ }^{\mathbf{R}_{0}} \mathbf{A}=\mathbf{A D}$ is the expression of matrix $\mathbf{A}$ in the base frame, where $\mathbf{D}$ is a transformation matrix, of which expression is given in [33].

For any prescribed trajectory $\mathbf{x}(t)$, the values of vector $\mathbf{q}_{a}$ can be found using the inverse kinematics and dynamics. Thus, taking into account that the manipulator is not in a Type 1 singularity [3], the terms $\mathbf{W}_{\mathbf{c}}$ and ${ }^{\mathbf{R}_{0}} \mathbf{W}_{\mathbf{p}}$ can be computed 
(using, or not, some recursive algorithm [34]). However, for a trajectory passing through a Type 2 singularity, the determinant of matrix ${ }^{\mathbf{R}_{0}} \mathbf{A}$ vanishes. Numerically, the values of the efforts applied by the actuators become infinite. In practice, the manipulator either is locked in such a position of the end-effector or it can not follow the prescribed trajectory.

As previously mentioned, in a Type 2 singularity, the determinant of matrix ${ }^{\mathbf{R}_{0}} \mathbf{A}$ vanishes. In other words, at least two of its columns are linearly dependant [33]. So, one may obtain such a relationship:

$$
\sum_{j=1}^{6} \alpha_{j} \mathbf{A}_{j}=\mathbf{0}
$$

where $\mathbf{A}_{j}$ represents the $j$-th column of matrix ${ }^{\mathbf{R}_{0}} \mathbf{A}$ and $\alpha_{j}$ are coefficients, which in general can be functions of $\mathbf{q}_{a}$ and $\mathbf{q}_{e}$. It should be noted that the vector $\mathbf{t}_{\mathrm{s}}=\left[\alpha_{1}, \alpha_{2}, \ldots, \alpha_{6}\right]^{T}$ represents the direction of the uncontrollable motion of the platform in a Type 2 singularity.

By substituting (6) into (4), we obtain

$$
\mathbf{A}_{j}^{T} \lambda=W_{j}, j=1, \ldots, 6
$$

where $W_{j}$ is the $j$-th row of vector ${ }^{\mathbf{R}_{0}} \mathbf{W}_{\mathbf{p}}$.

Then, from (6) and (7) the following conditions are derived:

$$
\sum_{j=1}^{6}\left(\alpha_{j} \mathbf{A}_{j}^{T} \lambda\right)=\sum_{j=1}^{6}\left(\alpha_{j} W_{j}\right)=0 .
$$

The right term of Eq. (8) corresponds to the scalar product of vectors $\mathbf{t}_{\mathrm{s}}$ and ${ }^{\mathbf{R}_{0}} \mathbf{W}_{\mathbf{p}}$.

Thus, in the presence of a Type 2 singularity, it is possible to satisfy conditions (8) if the wrench applied on the platform by the legs and external efforts ${ }^{R_{0}} W_{p}$ are orthogonal to the direction of the uncontrollable motion $\mathbf{t}_{\mathrm{s}}$. Otherwise, the dynamic model is not consistent. Obviously, in the presence of a Type 2 singularity, the displacement of the end-effector of the manipulator has to be planned to satisfy (8). Therefore, our task will be to achieve a trajectory which will allow the manipulator passing trough the Type 2 singularities, i.e. which will allow the manipulator respecting condition (8).

In the next section, an example illustrates the obtained results discussed above. This example presents a planar $5 R$ parallel manipulator.

\section{ILLUSTRATIVE EXAMPLE}

In the planar $5 R$ parallel manipulator, as shown in Fig. 1, the output axis is connected to the base by two legs, each of which consists of three revolute joints and two links. In each of the two legs, the revolute joint connected to the base is actuated. Thus, such a manipulator is able to position its output axis in a plane.

As shown in Fig. 1, the input joints are denoted as $A$ and $E$. The orientation of elements 1 and 4 are denoted $q_{1}^{e}$ and $q_{2}^{e}$, respectively. The common joint of the two legs is denoted as $C$, which is also the output axis with controlled parameters $x$ and $y$. A fixed global reference system $\boldsymbol{x} \boldsymbol{O} \boldsymbol{y}$ is located at the middle of segment $A E$ with the $\boldsymbol{y}$-axis normal to $\mathbf{A E}$ and the $\boldsymbol{x}$ axis directed along AE. The lengths of the links $A B, B C, C D$, $D E$ are respectively denoted as $L_{1}, L_{2}, L_{3}$ and $L_{4}$.

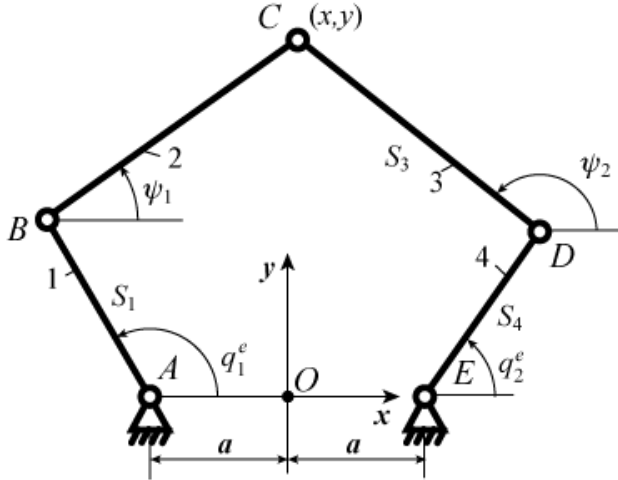

Figure 1. Kinematic chain of the planar $5 R$ parallel manipulator.

Actuators 1 and 2 are connected to links 1 and 4 , respectively, via Harmonic Drive ${ }^{\circledR}$ systems which are represented by a model similar to that given in [35]. The position of actuator $i$ is denoted as $q_{i}^{a}$. It is assumed that the actuator $i$ is capable to deliver a couple $\tau_{i}$ to the motor shaft, which is elastically coupled to the link $j$ of the robot $(i=1$ or $2, j=1$ or 4$)$. The flexibility of the drive system is modeled by a torsion spring with stiffness $k_{1}$. The gear ratio is denoted $n$. $I^{a}$ is the axial moment of inertia of the motor $i$ plus the Harmonic drive system.

In order to obtain relatively simple symbolic model for demonstrating the expected results, we propose to modelize the deformations of the robot by adding virtual joints on the links. We assume here that the link deformations of elements 2 and 3 are preponderant. Therefore, we add on them linear springs of stiffness $k_{2}$, that are directed along directions $\mathbf{B C}$ and $\mathbf{C D}$, respectively. The displacements of the spring linked to element 2 ( 3 , resp.) will be denoted as $\varepsilon_{1}\left(\varepsilon_{2}\right.$, resp.).

The singularity analysis of this manipulator [36] shows that the Type 2 singularities appear when links 2 and 3 are parallel (see also Fig. 2 in [1]). In both cases, the gained degree of freedom is an infinitesimal translation perpendicular to the links 2 and 3. However, if $L_{2}=L_{3}$, the gained degree of freedom may become a finite rotary motion.

Taking into account that the gravity is directed along $z$ axis (perpendicular to the plane of motions), the expression of the potential energy $V$ may be written as:

$$
V=0.5\left(k_{1}\left(\left(q_{1}^{e}-q_{1}^{a}\right)^{2}+\left(q_{2}^{e}-q_{2}^{a}\right)^{2}\right)+k_{2}\left(\varepsilon_{1}^{2}+\varepsilon_{2}^{2}\right)\right) .
$$

The expression of the kinetic energy is

$$
T=0.5\left(m\left(\dot{x}^{2}+\dot{y}^{2}\right)+I^{a}\left(\left(\dot{q}_{1}^{a}\right)^{2}+\left(\dot{q}_{2}^{a}\right)^{2}\right)\right),
$$

where $m$ is the mass of the payload and $\dot{x}$ and $\dot{y}$ are the velocities of point $C$ along $\boldsymbol{x}$ and $\boldsymbol{y}$ axes, respectively.

Thus the dynamic model can be obtained from (3) and (4):

$$
\begin{gathered}
k_{2}\left[\begin{array}{l}
\varepsilon_{1} \\
\varepsilon_{2}
\end{array}\right]+\mathbf{C}^{T} \mathbf{A}^{-T} \mathbf{W}_{\mathbf{p}}=\mathbf{0}, \mathbf{W}_{\mathbf{p}}=m\left[\begin{array}{c}
\ddot{x} \\
\ddot{y}
\end{array}\right] \\
k_{1}\left[\begin{array}{l}
q_{1}^{e}-q_{1}^{a} / n \\
q_{2}^{e}-q_{2}^{a} / n
\end{array}\right]+\mathbf{B}^{T} \mathbf{A}^{-T} \mathbf{W}_{\mathbf{p}}=\mathbf{0} \\
\tau=I^{a}\left[\begin{array}{l}
\ddot{q}_{1}^{a} \\
\ddot{q}_{2}^{a}
\end{array}\right]-\frac{k_{1}}{n}\left[\begin{array}{l}
q_{1}^{e}-q_{1}^{a} / n \\
q_{2}^{e}-q_{2}^{a} / n
\end{array}\right]
\end{gathered}
$$


Matrices A, B and $\mathbf{C}$ may be found from the loop closure equations:

$f_{1}=\left(x+a-L_{1} \cos q_{1}^{e}\right)^{2}+\left(y-L_{1} \sin q_{1}^{e}\right)^{2}-\left(L_{2}+\varepsilon_{1}\right)^{2}=0(12 \mathrm{a})$

$f_{2}=\left(x-a-L_{4} \cos q_{2}^{e}\right)^{2}+\left(y-L_{4} \sin q_{2}^{e}\right)^{2}-\left(L_{3}+\varepsilon_{2}\right)^{2}=0(12 \mathrm{~b})$

from which it comes:

$$
\begin{aligned}
\mathbf{A} & =\left[\frac{\partial f_{i}}{\partial \mathbf{x}}\right]=\left[\begin{array}{ll}
a_{11} & a_{12} \\
a_{21} & a_{22}
\end{array}\right] \\
& =2\left[\begin{array}{ll}
x-L_{1} \cos q_{1}^{e}+a & y-L_{1} \sin q_{1}^{e} \\
x-L_{4} \cos q_{2}^{e}-a & y-L_{4} \sin q_{2}^{e}
\end{array}\right] \\
\mathbf{B} & =-\left[\frac{\partial f_{i}}{\partial \mathbf{q}_{e}}\right] \\
& =-\left[\begin{array}{cc}
L_{1}\left(a_{11} \sin q_{1}^{e}-a_{12} \cos q_{1}^{e}\right) \\
0 & L_{4}\left(a_{21} \sin q_{2}^{e}-a_{22} \cos q_{2}^{e}\right)
\end{array}\right] \\
\mathbf{C} & =-\left[\frac{\partial f_{i}}{\partial \boldsymbol{\varepsilon}}\right]=2\left[\begin{array}{cc}
L_{2}+\varepsilon_{1} & 0 \\
0 & L_{3}+\varepsilon_{2}
\end{array}\right] \approx 2\left[\begin{array}{cc}
L_{2} & 0 \\
0 & L_{3}
\end{array}\right]
\end{aligned}
$$

with $\mathbf{x}=[x, y]^{T}, \mathbf{q}_{e}=\left[q_{1}^{e}, q_{2}^{e}\right]^{T}$ and $\boldsymbol{\varepsilon}=\left[\varepsilon_{1}, \varepsilon_{2}\right]^{T}$.

As the trajectory $\mathbf{x}(t)$ is known, from (11a), we can obtain the values of $\boldsymbol{\varepsilon}$. Introducing it into (12), the parameters $\mathbf{q}_{e}$ are computed. Finally, from (11b), the values of the actuated variables $\mathbf{q}_{a}$ are obtained. Differentiating it two times with respect to the time allows obtaining the values of the input torques.

Analyzing these expressions, it could be observed that the dynamic model depend on the position, velocity and acceleration of point $C$, but also of the jerk and its first derivative. Therefore, a ninth-degree polynomial has to be applied as a control law when the end-effector is not in singular configuration.

In order to avoid infinite values of the input torques when crossing a Type 2 singularity, Eq. (8) has to be satisfied. From matrix $\mathbf{A}$, one can find that the twist of the infinitesimal displacement in the singularity can be written under the form:

$$
\mathbf{t}_{\mathbf{s}}=\left[-\sin \psi_{1}, \cos \psi_{1}\right]^{T}
$$

Thus, the examined manipulator can pass through the given singular positions if the wrench $\mathbf{W}_{\mathbf{p}}$ determined by (11) is orthogonal to the direction of the uncontrollable motion $\mathbf{t}_{\mathrm{s}}$ described by (16).

Let us now consider the motion planning, which makes it possible to satisfy this condition. For this purpose the following parameters of manipulator's links are specified: $a=$ $0.2 \mathrm{~m}, L_{1}=L_{2}=L_{3}=L_{4}=0.25 \mathrm{~m} ; m=10 \mathrm{~kg} ; k_{1}=25000$ $\mathrm{Nm} / \mathrm{rad} ; k_{2}=350000 \mathrm{~N} / \mathrm{m}$. Moreover, we use the physical parameters of a real Harmonic Drive ${ }^{\circledR}$ system (HDUC-1U-11) coupled with an actuator Gamdrive (11-50-MB-SP-024-CR), that are $I^{a}=0.064 \cdot 10^{-4} \mathrm{~kg} \cdot \mathrm{m}^{2}$ and $n=50$.

With regard to the prescribed trajectory generation, the point $C$ should reproduce a motion along a straight line between the initial position $C_{0}\left(x_{0}, y_{0}\right)=C_{0}(0.1,0.345)$ and the final point $C_{f}\left(x_{f}, y_{f}\right)=C_{f}(-0.05,0.195)$ in $t_{f}=1.5 \mathrm{~s}$ (Fig. 2).

Thus, the given trajectory can be expressed as follows:

$$
\mathbf{x}=\left[\begin{array}{l}
x(t) \\
y(t)
\end{array}\right]=\left[\begin{array}{l}
x_{0}+s(t)\left(x_{f}-x_{0}\right) \\
y_{0}+s(t)\left(y_{f}-y_{0}\right)
\end{array}\right]
$$

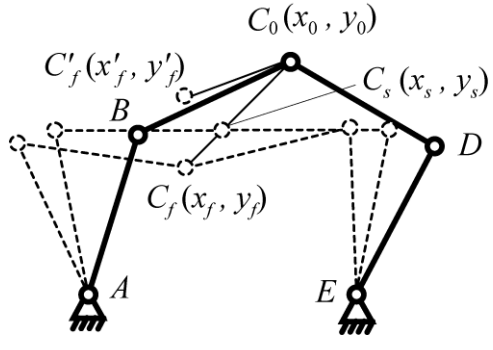

Figure 2. Initial, singular and final positions of the planar $5 \mathrm{R}$ parallel manipulator.

However, the manipulator will pass by a Type 2 singular position at point $C_{s}\left(x_{s}, y_{s}\right)=C_{s}(0,0.245)$ (Fig. 2).

Developing the condition (8) for passing through the singular position for the planar $5 R$ parallel manipulator at point $C_{s}$, we obtain:

$$
\ddot{y}=0
$$

Then, taking into account that the velocity and the acceleration of the end-effector in initial and final positions are equal to zero, the following thirteen boundary conditions are found:

$$
\begin{gathered}
s\left(t_{0}\right)=0, \\
s\left(t_{f}\right)=1, \\
s\left(t_{s}=1 \mathrm{~s}\right)=2 / 3, \\
\dot{s}\left(t_{0}\right)=0, \\
\dot{s}\left(t_{f}\right)=0, \\
\dot{s}\left(t_{s}\right)>0\left(\text { in our case } \dot{s}\left(t_{s}\right)=1.5\right), \\
\ddot{s}\left(t_{0}\right)=0, \\
\ddot{s}\left(t_{f}\right)=0, \\
\ddot{s}\left(t_{s}\right)=0 . \\
\dddot{s}\left(t_{0}\right)=0, \\
\dddot{s}\left(t_{f}\right)=0, \\
d\left(\dddot{s}\left(t_{0}\right)\right) / d t=0, \\
d\left(\dddot{s}\left(t_{f}\right)\right) / d t=0,
\end{gathered}
$$

From (19)-(31), the following twelfth order polynomial trajectory planning is found:

$$
\begin{aligned}
s(t)= & 14.96 t^{5}-6.38 t^{6}-128.38 t^{7}+334.66 t^{8} \\
& -381.71 t^{9}+230.08 t^{10}-71.67 t^{11}+9.12 t^{12}
\end{aligned}
$$

Thus the generation of the motion by the obtained twelfth order polynomial makes it possible to pass through the singularity without perturbation and the input torques remain in the limits of finite values.

In order to compare the different cases of trajectory planning, in Figs. 3 and 4 are given the values of the input torques obtained using the software ADAMS for the following numerical simulations:

A: a trajectory between points $C_{0}$ and $C_{f}^{\prime}\left(x_{f}^{\prime}, y_{f}^{\prime}\right)=C_{f}^{\prime}(-$ $0.05,0.295$ ) (Fig. 2) without meeting any singularity. For such a case, the following ninth order polynomial law is used $s(t)=16.59 t^{5}-36.87 t^{6}+31.6 t^{7}-12.29 t^{8}+1.82 t^{9}$ for the trajectory planning out of the singular zone of the manipulator. In this case the values of the input torques are finite. 


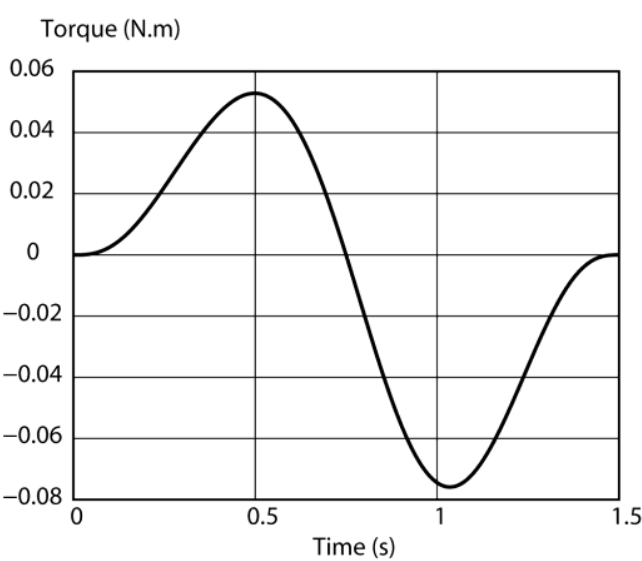

(a) Case A

Torque (N.m)

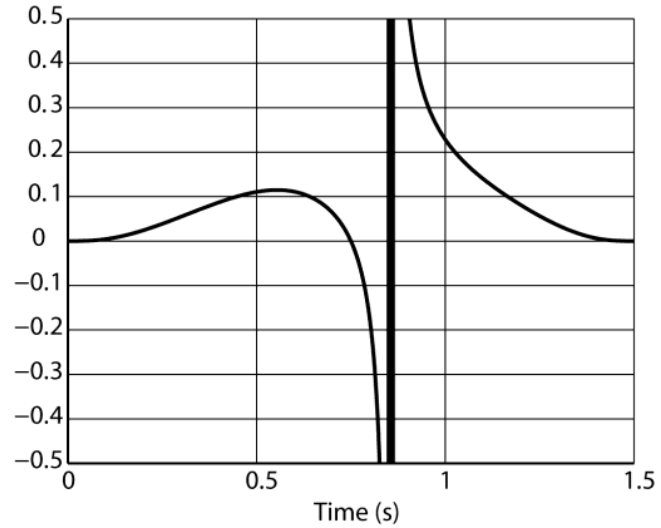

(b) Case B

Torque (N.m)

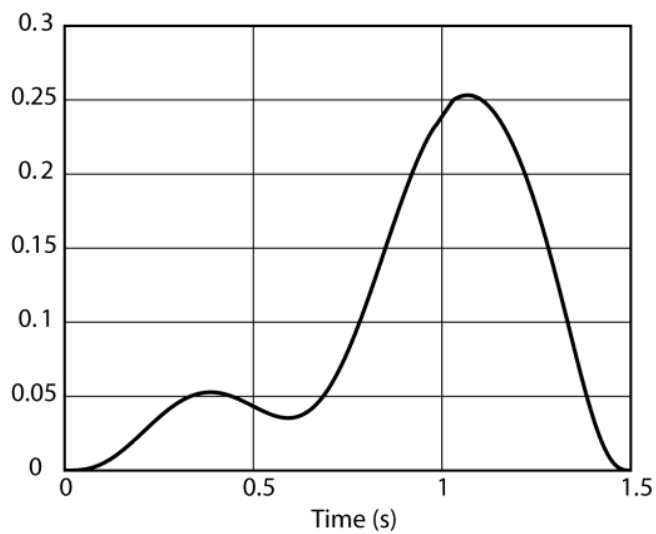

(c) Case C

Figure 3. Torques values for the actuator 1 .

B: $\quad$ the ninth order polynomial law $s(t)=16.59 t^{5}-36.87 t^{6}+$ $31.6 t^{7}-12.29 t^{8}+1.82 t^{9}$ for the trajectory planning between $C_{0}$ and $C_{f}$ inside the singular zone for the manipulator. In this case the values of the input torques close to the singular positions tend to infinity.

C: the twelfth order polynomial law of Eq. (32) for the trajectory planning of the manipulator inside the singular zone. The obtained results show that the values of the input torques are finite.

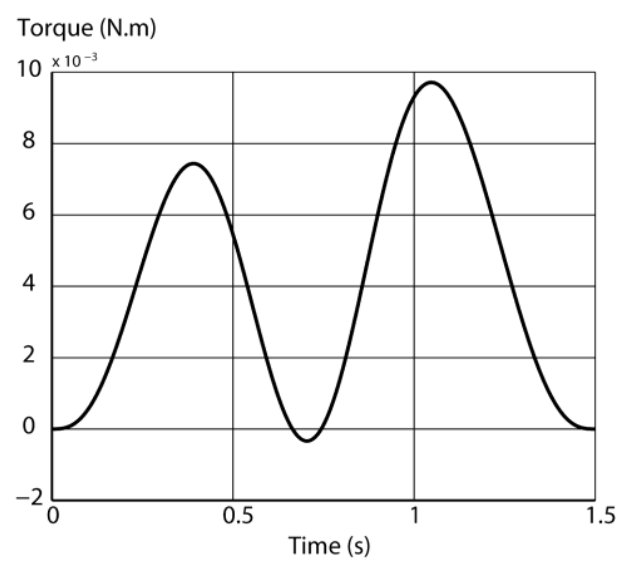

(a) Case A

Torque (N.m)

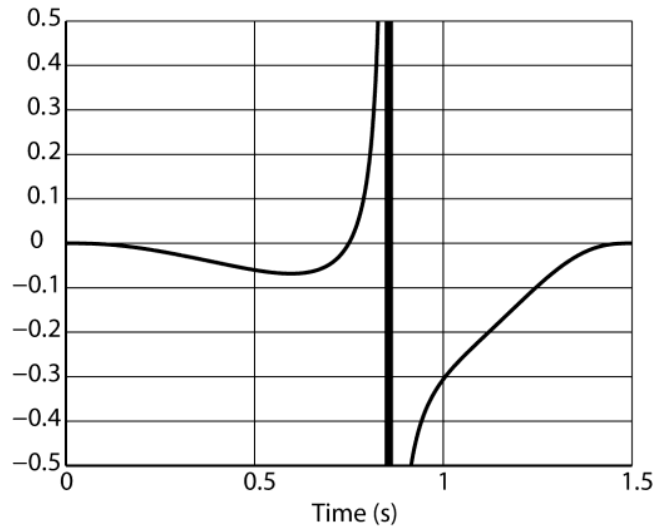

(b) Case B

Torque (N.m)

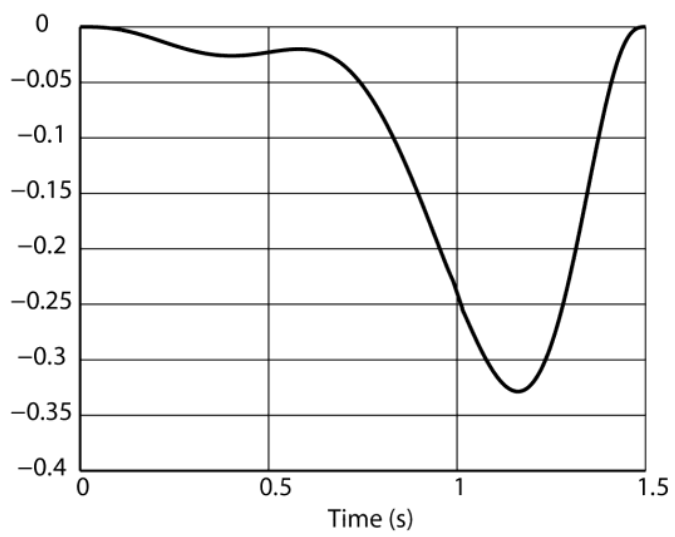

(c) Case C

Figure 4. Torques values for the actuator 2.

Thus, the numerical simulations show that the obtained optimal dynamic conditions assume the passing of the flexible manipulator through the singular position.

\section{CONCLUSION}

In our previous work, we have shown that any parallel manipulator can pass through the singular positions without perturbation of motion if the wrench applied on the endeffector by the legs and external efforts is orthogonal to the twist along the direction of the uncontrollable motion [1]. This 
condition was applied to the rigid-link manipulators. The obtained results showed that the planning of motion for assuming the optimal force generation can be carried out by a eight order polynomial law. In our other study [2] the rigidlink flexible-joint manipulators have been studied. It was shown that the degree of the polynomial law should be different, when the flexibility of actuated joints is introduced. The obtained results disclosed that the planning of motion for assuming the optimal force generation in the rigid-link flexible-joint manipulators must be carried out by a twelfth order polynomial law.

In this paper, we have expanded the information about the dynamic properties of parallel manipulators in the presence of Type 2 singularity by including in the studied problem the link flexibility and the payload. The obtained results have shown that the planning of motion for assuming the optimal force generation in the structurally flexible manipulators with payload must be also carried out by a twelfth order polynomial law.

The suggested technique was illustrated by a $5 R$ planar parallel manipulator. The obtained dynamic properties have been validated by numerical simulations carried out using the software ADAMS.

\section{REFERENCES}

[1] Briot, S., and Arakelian, V., 2008, "Optimal Force Generation in Parallel Manipulators for Passing through the Singular Positions," International Journal of Robotics Research, Vol. 27 (8), pp. 967-983.

[2] Briot, S., and Arakelian, V., 2010, "On the Dynamic Properties of Rigid-Link Flexible-Joint Parallel Manipulators in the Presence of Type 2 Singularities," ASME Journal of Mechanisms and Robotics, Vol. 2 (2).

[3] Gosselin, C.M., and Angeles, J., 1990, "Singularity Analysis of Closed-Loop Kinematic Chains," IEEE Transactions on Robotics and Automatics, Vol. 6(3), pp. 281-290.

[4] Zlatanov, D., Fenton, R.G., Benhabib, B., 1994, "Singularity Analysis of Mechanisms and Robots via a Velocity-Equation Model of the Instantaneous Kinematics," Proceedings of the 1994 IEEE International Conference on Robotics and Automation, Vol. 2, pp. 980-991.

[5] Zein, M., Wenger, P., and Chablat, D., 2007, "Singular Curves in the Joint Space and Cusp Points of 3-RPR parallel manipulators", Robotica, Vol. 25(6), pp. 717724.

[6] Zein, M., Wenger, P. and Chablat, D., 2008, "NonSingular Assembly-mode Changing Motions for 3-RPR Parallel Manipulators", Mechanism and Machine Theory, Vol. 43(4), pp. 480-490.

[7] Kanaan D., Wenger P., Caro S., and Chablat D., 2009, "Singularity Analysis of Lower-Mobility Parallel Manipulators Using Grassmann-Cayley Algebra", IEEE Transactions on Robotics, Vol. 25(5), pp. 995-1004.

[8] Zlatanov, D., Bonev, I.A., and Gosselin, C. M., 2002, "Con-straint Singularities of Parallel Mechanisms," IEEE International Conference on Robotics and Automation (ICRA 2002), Washington, D.C., USA, May 11-15.
[9] Hunt, K.H., 1987, "Special Configurations of RobotArms via Screw Theory," Robotica, Vol. 5, pp. 17-22.

[10] Merlet, J.-P., 1989, "Singular Configurations of Parallel Manipulators and Grassmann Geometry," The International Journal of Robotics Research, Vol. 8(5), pp. 45-56.

[11] Bonev, I.A., Zlatanov, D., and Gosselin, C.M., 2003, "Singularity Analysis of 3-DOF Planar Parallel Mechanisms via Screw Theory," ASME Journal of Mechanical Design, Vol. 125(3), pp. 573-581.

[12] Briot, S. and Arakelian, V., 2007, "Singularity Analysis of PAMINSA Manipulator," The 12th World Congress in Mechanism and Machine Science, June 18-21, Besançon, France.

[13] Gosselin, C.M., 1992, "The Optimum Design of Robotic Manipulators using Dexterity Indices," Robotics and Autonomous Systems, Vol. 9(4), pp. 213-226.

[14] Rakotomanga, N., Chablat, D., and Caro, S., 2008, "Kinetostatic Performance of a Planar Parallel Mechanism with Variable Actuation", 11th International Symposium on Advances in Robot Kinematics, Kluwer Academic Publishers, Batz-sur-mer, France, June.

[15] Merlet, J.-P., 2006, "Jacobian, Manipulability, Condition Number, and Accuracy of Parallel Robots," ASME Journal of Mechanical Design, Vol. 128(1), pp. 199-206.

[16] Angeles, J., 2007, "Fundamentals of Robotic Mechanical Systems: Theory, Methods, and Algorithms," 3rd edition, Springer, New York.

[17] Alba-Gomez, O., Wenger, P., and Pamanes, A., 2005, "Consistent Kinetostatic Indices for Planar 3-DOF Parallel Manipulators, Application to the Optimal Kinematic Inversion," Proc. ASME 2005 IDETC/CIE Conference, Long Beach, California, September 24-28.

[18] Arakelian, V., Briot, S., and Glazunov, V., 2008, "Increase of Singularity-Free Zones in the Workspace of Parallel Manipulators using Mechanisms of Variable Structure," Mechanism and Machine Theory, Vol. 43(9), pp. 1129-1140.

[19] Hubert, J., and Merlet, J.-P., 2008, "Singularity Analysis through Static Analysis," Advances in Robot Kinematics, Springer, pp.13-20.

[20] Alvan, K., and Slousch, A., 2003, "On the Control of the Spatial Parallel Manipulators with Several Degrees of Freedom," Mechanism and Machine Theory, SaintPetersburg, Vol. 1, pp. 63-69.

[21] Dasgupta, B., and Mruthyunjaya, T., 1998, "Force Redundancy in Parallel Manipulators: Theoretical and Practical Issues," Mechanism and Machine Theory, Vol. 33(6), pp.724-742.

[22] Glazunov, V., Kraynev, A., Bykov, R., Rashoyan, G., and Novikova, N., 2004, "Parallel Manipulator Control While Intersecting Singular Zones," Proc. 15th Symposium on Theory and Practice of Robots and Manipulators (RoManSy) CISM-IFToMM, Montreal.

[23] Kotlarski, J., Do Thanh, T., Abdellatif, H., and Heimann, B., 2008 "Singularity Avoidance of a Kinematically Redundant Parallel Robot by a Constrained Optimization of the Actuation Forces," Proceedings of the Seventeenth CISM-IFToMM Symposium RoManSy, pp.435-442. 
[24] Hesselbach, J., Wrege, J., Raatz A., and Becker, O., 2004, "Aspects on the Design of High Precision Parallel Robots," Assembly Automation, Vol. 24(1), pp. 49-57.

[25] Bhattacharya, S., Hatwal, H., and Ghosh, A., 1998, "Comparison of an Exact and Approximate Method of Singularity Avoidance in Platform Type Parallel Manipulators," Mechanism and Machine Theory, Vol. 33(7), pp. 965-974.

[26] Dasgupta, B., and Mruthyunjaya, T., 1998, "SingularityFree Path Planning for the Steward Platform Manipulator," Mechanism and Machine Theory, Vol. 33(6), pp. 715-725.

[27] Kemal Ider, S., 2005, "Inverse Dynamics of Parallel Manipulators in the Presence of Drive Singularities," Mechanism and Machine Theory, Vol. 40, pp. 33-44.

[28] Kevin Jui, C.K., and Sun, Q., 2005, "Path Tracking of Parallel Manipulators in the Presence of Force Singularity," ASME Journal of Dynamic Systems, Measurement and Control, Vol. 127, pp. 550-563.

[29] Nenchev, D.N., Bhattacharya, S., and Uchiyama, M., 1997, "Dynamic Analysis of Parallel Manipulators under the Singularity-Consistent Parameterization," Robotica, Vol. 15(4), pp. 375-384.

[30] Perng, M.H., and Hsiao, L., 1999, "Inverse Kinematic Solutions for a Fully Parallel Robot with Singularity Robustness," The International Journal of Robotics Research, Vol. 18(6), pp. 575-583.

[31] Bouzgarrou, B.C., Ray, P., and Gogu, G., 2005, "New Approach for Dynamic Modelling of Flexible Manipulators," Proc. IMechE, Part K: Journal of Multibody Dynamics, Vol. 219, pp. 285-298.

[32] Khalil, W., and Guégan, S., 2002, “A Novel So-lution for the Dynamic Modeling of Gough-Stewart Manipulators," Proc. IEEE International Conference on Robotics and Automation, Washington DC., USA, May 11-15.

[33] Merlet, J.-P., 2006, "Parallel Robots," Springer, 2nd edition.

[34] Boyer, F., and Khalil, W., 1996, "Simulation of Flexible Manipulators Using Newton-Euler Inverse Dynamic Model," Proceedings of the 1996 IEEE International Conference on Robotics and Automation, Minneapolis, Minnesota, April.

[35] Spong, M.W., Khorasani, K., and Kokotovic, P.V., 1987, "An Integral Manifold Approach to the Feedback Control of Flexible Joint Robots," IEEE Journal of Robotics and Automation, Vol. 3(4), pp. 291-300.

[36] Liu, X.-J., Wang, J., and Pritschow, G., 2006, "Kinematics, Singularity and Workspace of Planar 5R Symmetrical Parallel Mechanism," Mechanism and Machine Theory, Vol. 41(2), pp. 119-144. 